\title{
The effect of continuous positive airway pressure on pulmonary function may depend on the basal level of forced expiratory volume in 1 second
}

\author{
Annia Schreiber ${ }^{1}$, Sara Surbone ${ }^{2}$, Alberto Malovini ${ }^{3}$, Marco Mancini $^{4}$, Francesca Cemmi ${ }^{5}$, \\ Giancarlo Piaggi ${ }^{1}$, Piero Ceriana ${ }^{1}$, Annalisa Carlucci ${ }^{1}$
}

${ }^{1}$ Pulmonary Rehabilitation Unit, Istituti Clinici Scientifici Maugeri, Pavia, Italy; ${ }^{2}$ Unità Operativa di Pneumologia, Ospedale Asilo Vittoria di Mortara, Azienda Socio Sanitaria Territoriale di Pavia, Italy; ${ }^{3}$ Laboratory of Informatics and Systems Engineering for Clinical Research, ${ }^{4}$ Direzione Sanitaria, Istituti Clinici Scientifici Maugeri, Pavia, Italy; ${ }^{5}$ Unità Operativa di Pneumologia, Ospedale Pederzoli, Peschiera del Garda (Verona), Italy Contributions: (I) Conception and design: A Schreiber, M Mancini, A Carlucci, S Surbone, F Cemmi; (II) Administrative support: P Ceriana, M Mancini; (III) Provision of study materials or patients: A Schreiber, S Surbone, M Mancini, F Cemmi, A Carlucci; (IV) Collection and assembly of data: A Schreiber, S Surbone, F Cemmi, A Carlucci; (V) Data analysis and interpretation: A Schreiber, A Malovini, A Carlucci; (VI) Manuscript writing: All authors; (VII) Final approval of manuscript: All authors.

Correspondence to: Annia Schreiber, MD. Pulmonary Rehabilitation Unit, Istituti Clinici Scientifici Maugeri, Via Salvatore Maugeri 10, 27100 Pavia, Italy. Email: annia.schreiber@icloud.com.

Background: The coexistence of chronic obstructive pulmonary disease (COPD) and obstructive sleep apnea (OSA), also referred to as overlap syndrome (OS), is associated with a high rate of morbidity, COPD exacerbations and mortality. Treatment with continuous positive airway pressure (CPAP) has proven to significantly decrease the rate of these complications. However, data concerning the effect of CPAP on pulmonary function are scarce and conflicting. The aim of our study was to evaluate the effect of 1 year of CPAP treatment on arterial blood gases (ABGs) and pulmonary function tests in patients with OS and its potential relationship with the baseline severity of airway obstruction. A secondary aim was to search for predictors of changes in the evaluated parameters.

Methods: A retrospective study on a cohort of 92 patients (74 males) discharged from the Pulmonary Rehabilitation Unit of the Istituti Clinici Scientifici Maugeri in Pavia (Italy) from January 2013 to January 2016, with a diagnosis of OS and a prescription of CPAP, was conducted. Collected data at discharge were compared with 1-year follow-up data.

Results: After 1 year of CPAP, we observed the following: (I) a significant improvement in ABGs in all patients [median $\mathrm{pO}_{2} 65.0$ (59.0-70.0) vs. 71 (64.8-77.1) mmHg, $\mathrm{pCO}_{2} 39.8$ (36.2-43.5) vs. 38.3 (32.3-44.2) at baseline and after 1 year respectively, $\mathrm{P}<0.001]$, which was more pronounced in patients who were hypercapnic at baseline; (II) no significant change in respiratory function in the whole population; (III) a significant change in forced expiratory volume in 1 second $\left(\mathrm{FEV}_{1}\right)$ only under and above a threshold of $79.1 \%$ of basal $\mathrm{FEV}_{1}$ with an opposite trend. In particular, patients with a basal $\mathrm{FEV}_{1}$ below that threshold significantly improved [median $\mathrm{FEV}_{1} 70$ (-70 to 200) $\mathrm{mL}, \mathrm{P}=0.001$ ], whereas patients with a basal $\mathrm{FEV}_{1}$ above the same threshold significantly worsened [median $\mathrm{FEV}_{1}-270(-370$ to -130$) \mathrm{mL}, \mathrm{P}=3.05 \times 10^{-5}$ ].

Conclusions: A population of overlap patients treated with CPAP may experience a different change in airflow obstruction after 1 year depending on the severity of baseline obstruction.

Keywords: Overlap syndrome (OS); obstructive sleep apnea (OSA); chronic obstructive pulmonary disease (COPD); continuous positive airway pressure (CPAP); pulmonary function tests

Submitted Jan 17, 2018. Accepted for publication Oct 16, 2018.

doi: $10.21037 /$ jtd.2018.10.103

View this article at: http://dx.doi.org/10.21037/jtd.2018.10.103 


\section{Introduction}

Chronic obstructive pulmonary disease (COPD) and obstructive sleep apnea (OSA) represent two of the most prevalent chronic respiratory disorders, affecting respectively $10 \%$ and $5 \%$ of the adult population (1).

The health burden associated with the coexistence of both disorders, also referred to as overlap syndrome (OS) (2), is far greater than the sum of the two components in terms of morbidity, incidence of COPD exacerbations and mortality (2-4).

Treatment with continuous positive airway pressure (CPAP) has proven to significantly decrease the rate of COPD exacerbations and mortality in patients with OS, lowering the risk of both conditions to levels associated with COPD alone (5). Notably, less is known about the effect of CPAP treatment on pulmonary function, and the scarce existing data are conflicting $(6,7)$. Furthermore, studies on this topic are small and, consequently, their results are inconclusive.

A previous study documented a deterioration in forced expiratory volume in 1 second $\left(\mathrm{FEV}_{1}\right)$ in the 2 years following the introduction of CPAP therapy (6) with a linear correlation between the average hours of daily CPAP use and the percent decrease in $\mathrm{FEV}_{1}$. Another study (8) found significant improvements in arterial blood gases (ABGs) and pulmonary function tests at 6 and 18 months after the beginning of CPAP therapy. Finally, Toraldo and colleagues (7), in line with the last reported study, demonstrated a significant increase in $\mathrm{FEV}_{1}$ and $\mathrm{FEV}_{1}$ / forced vital capacity (FVC) ratio compared to baseline values even after 24 months of CPAP treatment.

The primary aim of our study was to evaluate the effect of 1 year of CPAP treatment on ABGs and respiratory function tests in a series of patients with OS and to relate the treatment results to the severity of baseline airway obstruction.

The secondary aim was to search for predictors of changes in the evaluated parameters.

\section{Methods}

\section{Patients}

We retrospectively collected data of patients admitted to the Pulmonary Rehabilitation Unit of the Istituti Clinici Scientifici Maugeri in Pavia (Italy) from January 2013 to January 2016 who met all of the following criteria: a diagnosis of overlap between COPD and OSA, clinical stability for at least 3-4 weeks (as assessed by the absence of any worsening of symptoms and stability in ABGs (no acidosis, defined as $\mathrm{pH} \leq 7.35$ ), a prescription of domiciliary CPAP, and a follow-up in our hospital for at least 1 year after CPAP prescription.

Patients were excluded from the study if they had other sleep or respiratory disorders (e.g., resless leg syndrome, insomnia), an average use $<4$ hours per night (assessed 3 months after CPAP prescription by the analysis of the memory card of the ventilator) and if 1-year follow-up data were lacking or incomplete. Other exclusion criteria were major uncontrolled medical or psychiatric conditions; cancer; surgery of the upper airways, nose, sinuses or middle ears in the 3 months before enrollment; signs or symptoms of acute exacerbation of COPD; or an $\mathrm{ABG} \mathrm{pH} \leq 7.35$.

\section{Measurements}

Spirometry and lung volumes were measured with the subjects sitting in a body plethysmograph following the American Thoracic Society/European Respiratory Society (ATS/ERS) recommendations $(9,10)$. The reference values reported in the Gold Guidelines were used (11). The diagnosis and severity of COPD were established if an irreversible obstructive functional defect $\left(\mathrm{FEV}_{1} / \mathrm{FVC}\right.$ ratio $\leq 70 \%$ ) together with a past history of cigarette smoking were present, in accordance with the Global Initiative for Chronic Obstructive Lung Disease (GOLD) (11).

ABG samples were taken from the radial artery with the spontaneously breathing awake patient in the sitting position. The samples were analyzed with a blood gas analyzer (ABL 520-Radiometer, Copenhagen, Denmark).

A diagnosis of OSA was made according to standard criteria (12): all patients underwent standard sleep evaluation and full polysomnography, analyzed according to the American Academy of Sleep Medicine (AASM) 2007 Guidelines (13). A diagnosis of OS was made when COPD patients presented clinical symptoms and an apneahypopnea index (AHI) of 10 or more.

After the diagnosis of OSA, patients received CPAP adaptation and treatment. A full-night manual CPAP titration in a laboratory was performed according to AASM Guidelines (14) to identify the best level of pressure to treat the sleep disorder.

Treatment efficacy was confirmed by a further overnight polygraphy and, once a good level of tolerance was reached (defined as an average use of at least four hours/night, as indicated by the memory card of the device), a final 
Table 1 Cohort characterization: demographic and clinical variables at baseline

\begin{tabular}{|c|c|}
\hline Patients ( $n=92)$ & Values \\
\hline Age (years) & $66(60.2-74.0)$ \\
\hline Males, n [\%] & $74[80.4]$ \\
\hline BMI $\left(\mathrm{kg} / \mathrm{m}^{2}\right)$ & $31.9(28.2-37.1)$ \\
\hline \multicolumn{2}{|l|}{ Comorbidities, n [\%] } \\
\hline Presence $>2$ & 39 [42] \\
\hline Arterial hypertension & $70[76]$ \\
\hline Cardiopathy & $28[30]$ \\
\hline Renal failure & 8 [9] \\
\hline Diabetes & 19 [21] \\
\hline Cancer & $4[4]$ \\
\hline Pulmonary hypertension & $15[16]$ \\
\hline \multicolumn{2}{|l|}{ Functional tests } \\
\hline $\mathrm{FEV}_{1}(\mathrm{~L})$ & $1.58(1.2-2.1)$ \\
\hline $\mathrm{FEV}_{1}(\%$ of predicted value) & $62.1(46.8-79.5)$ \\
\hline VC (L) & $2.8(2.2-3.5)$ \\
\hline VC (\% of predicted value) & $86(69.0-99.0)$ \\
\hline $\mathrm{FEV}_{1} / \mathrm{FVC}(\%)$ & $61(49.0-65.0)$ \\
\hline TLC (\%) & $85( \pm 18)$ \\
\hline \multicolumn{2}{|l|}{ ABGs } \\
\hline $\mathrm{PaO}_{2}(\mathrm{mmHg})$ & $65.0(59.0-70.0)$ \\
\hline $\mathrm{PaCO}_{2}(\mathrm{mmHg})$ & $39.8(36.2-43.5)$ \\
\hline $\mathrm{pH}$ & $7.43(7.41-7.45)$ \\
\hline \multicolumn{2}{|l|}{ Full polysomnography } \\
\hline AHI (events/hours) & $43.8(28.7-65.5)$ \\
\hline $\mathrm{SaO}_{2}$ mean $(\%)$ & $91.6(89.0-93.2)$ \\
\hline ODI (events/hours) & $34.2(22.2-55.8)$ \\
\hline $\mathrm{T}_{90}(\%)$ & $17.8(5.0-71.0)$ \\
\hline
\end{tabular}

Distribution: for categorical variables, the absolute count (frequency, \%) or median (25th-75th percentiles) of the distribution is reported. $\mathrm{FEV}_{1}$, forced expiratory volume in 1 second; FVC, forced vital capacity; VC, vital capacity; TLC, total lung capacity; $\mathrm{AHI}$, apnea-hypopnea index; ODI, oxygen desaturation index; $\mathrm{T}_{90}$, percentage of sleep time with $\mathrm{SaO}_{2}$ below $90 \%$.

prescription of treatment was made. In accordance with the Italian health system, when a domiciliary CPAP is prescribed, the policy is to rotate among different models and manufacturers. All of these models are invariably equipped with software that enables us to record and analyze data.

Patients were followed up 3 months later to check for adherence to the therapy and possible technical or clinical problems. One year later, a full polysomnography, as well as pulmonary functional tests and $\mathrm{ABG}$ measurements, were repeated.

Patients with a more severe functional defect received an intermediate pulmonary function test evaluation 6 months after CPAP prescription and discharge. This intermediate test was not considered in this study.

\section{Study design}

Demographic data, functional tests, ABGs, full polysomnography, comorbidities (1 or more among arterial hypertension, ischemic cardiopathy and arrhythmia, diabetes and renal failure) recorded at baseline and 1 year later were collected.

The study was approved by the Ethics Committee of the Istituti Clinici Scientifici Maugeri (Number 844 CEC).

\section{Statistical analysis}

The distributions of categorical variables were described by counts and frequencies (\%). Quantitative variable distributions were described by the median (25th-75th percentiles). The presence of statistically significant differences in terms of quantitative variables characterized by a continuous distribution between binary independent variables was tested by the Wilcoxon rank sum test. The presence of statistically significant variations in terms of quantitative variables between time points was tested by the Wilcoxon signed rank test. The association between categorical variables was tested either by Fisher's exact test or Pearson's chi-squared test with Yates' continuity correction as appropriate. Multivariate logistic regression coupled with a stepwise features selection procedure was used to identify the most informative set of variables associated with binary outcomes. Informative thresholds were identified by a classification tree-based approach. Linear regression was used to estimate the correlation between quantitative variables and to quantify the impact of potential confounders. The significance threshold was set at $\mathrm{P}<0.05$. All statistical analyses were performed using $\mathrm{R}$ statistical Software (www.r-project.org).

\section{Results}

A total of 92 patients ( 74 males) who were hospitalized during the observation period met our inclusion criteria. The median age was 66 (60.2-74.0). Patients' demographic 
Table 2 Results from univariate association tests

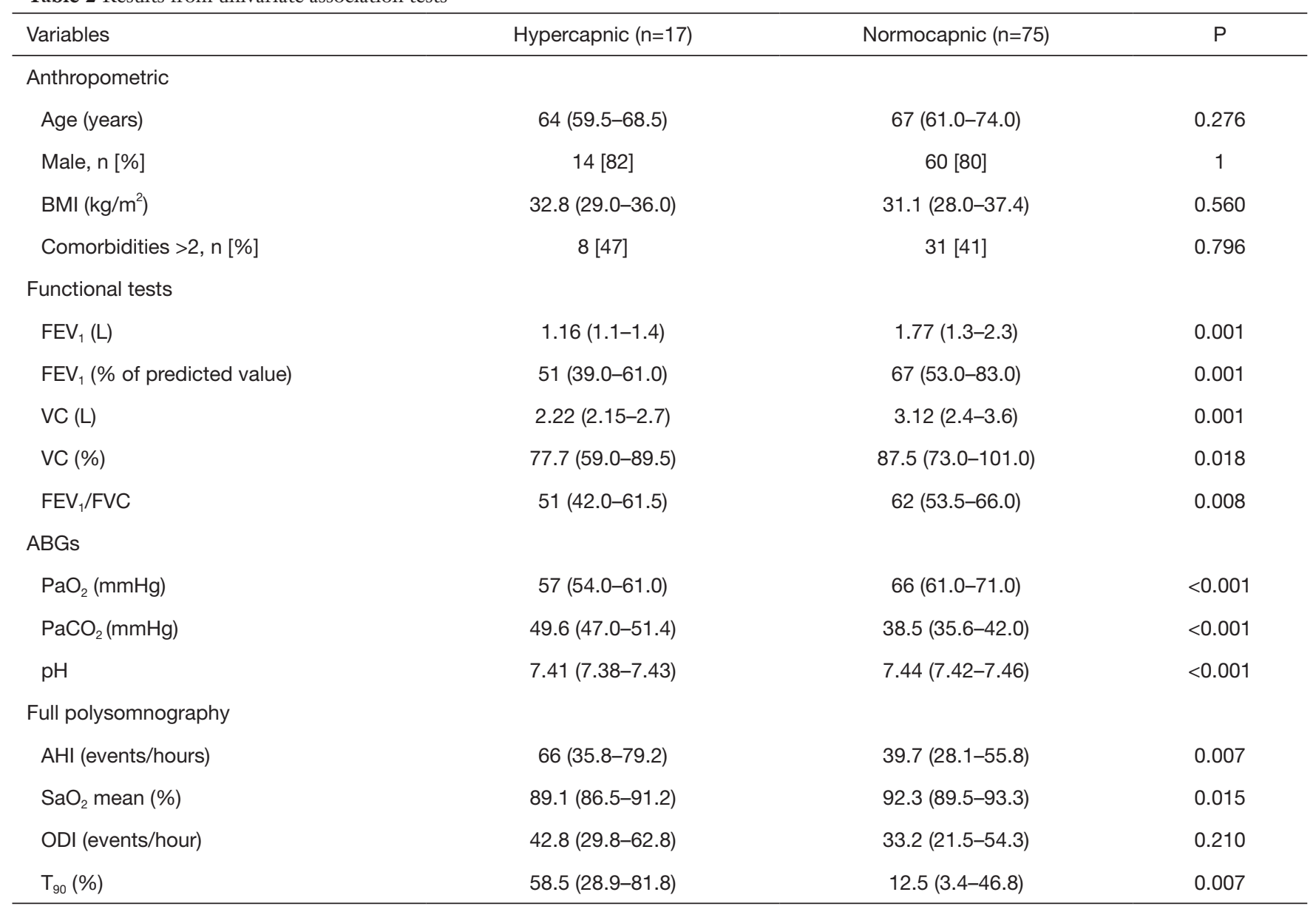

For categorical variables, the absolute count (frequency, \%) or median (25th-75th percentiles) of the distribution estimated in hypercapnic and normocapnic patients is reported; a $\mathrm{P}$ value from the Fisher's exact test or from the Wilcoxon Rank Sum test was considered significant when $<0.05$. $\mathrm{FEV}_{1}$, forced expiratory volume in 1 second; FVC, forced vital capacity; VC, vital capacity; AHI, apnea-hypopnea index; ODI, oxygen desaturation index; $\mathrm{T}_{90}$, percentage of sleep time with $\mathrm{SaO}_{2}$ below $90 \%$.

and clinical data at baseline are reported in Table 1. The median $\mathrm{FEV}_{1} \%$ value was $62.1 \%$ (46.8-79.5\%). According to the GOLD classification, 23 patients $(25.0 \%)$ were distributed in class 1, $43(46.7 \%)$ in class 2, $20(21.7 \%)$ in class 3 and $6(6.5 \%)$ in class 4.

The median AHI was 43.8 (28.7-65.5) events per hour with a median nocturnal saturation of $91.6 \%(89.0-93.2 \%)$; 17 of 92 patients $\left(18.47 \%\right.$ ) showed a $\mathrm{pCO}_{2}>45 \mathrm{mmHg}$.

A comparison between hypercapnic and normocapnic patients showed that the two populations were not statistically different in terms of anthropometric variables and comorbidities. However, hypercapnic patients had more severe airway obstruction, more severe hypoxemia at baseline and worse AHI and nocturnal gas exchanges, as recorded during polysomnography (Table 2).
A multivariate logistic regression analysis showed that AHI [OR $=1.08(1.02-1.16), \mathrm{P}=0.012$ ], the percentage of sleep time with $\mathrm{SaO}_{2}$ below $90 \%\left(\mathrm{~T}_{90}\right)$ [OR $=1.03(1.01-$ 1.06), $\mathrm{P}=0.029]$, vital capacity $(\mathrm{VC})[\mathrm{OR}=0.20(0.05-0.57)$, $\mathrm{P}=0.008]$ and $\mathrm{FEV}_{1} / \mathrm{FVC}[\mathrm{OR}=0.91(0.83-0.97), \mathrm{P}=0.01]$ at baseline were the strongest predictors of hypercapnia. Baseline characteristics (age, gender and comorbidities) did not significantly influence the probability of being hypercapnic $(\mathrm{P}>0.30)$; therefore, these were not included in the multivariate model.

After 1 year of treatment, we observed a significant improvement in $\mathrm{pO}_{2}$ [median value 65.0 (59.0-70.0) vs. 71 (64.8-77.1) $\mathrm{mmHg}$, at baseline and after 1 year, respectively, $\left.\mathrm{P}=5.6 \times 10^{-9}\right]$ and $\mathrm{pCO}_{2}[39.8(36.2-43.5)$ vs. 38.3 (32.3-44.2) $\mathrm{mmHg}$ respectively, $\mathrm{P}=0.0001]$ in the general 

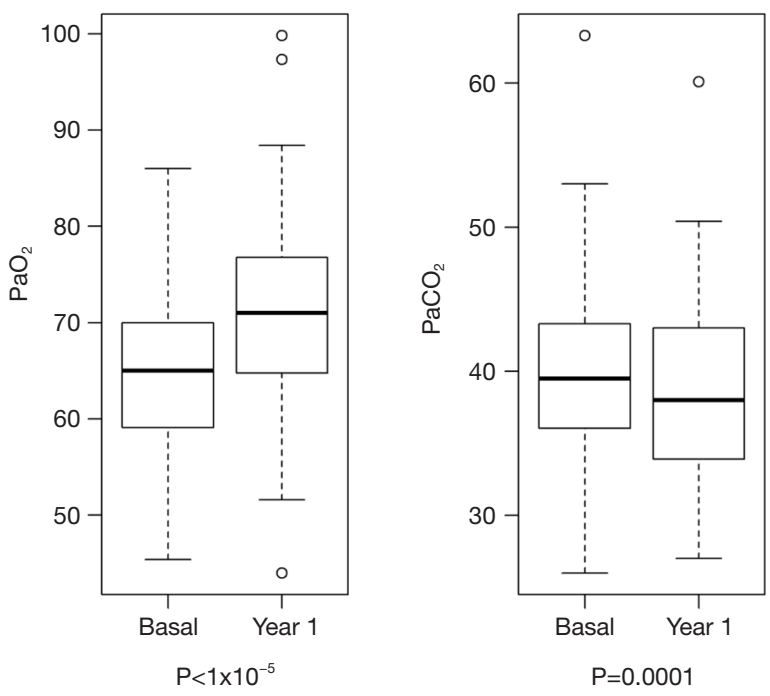

Figure 1 ABGs at baseline and after 1 year in the whole patient population. After 1 year of treatment, we observed a significant improvement in $\mathrm{pO}_{2}$ [median value 65.0 (59.0-70.0) vs. 71 (64.8$77.1) \mathrm{mmHg}$ at baseline and after 1 year, respectively, $\left.\mathrm{P}=5.6 \times 10^{-9}\right]$ and $\mathrm{pCO}_{2}$ [39.8 (36.2-43.5) vs. 38.3 (32.3-44.2) mmHg respectively, $\mathrm{P}=0.0001]$ in the general population.

population (Figure 1).

Both improvements appeared significantly greater in the subgroup of hypercapnic patients compared to the normocapnic ones [median $\mathrm{pO}_{2} 57$ (54-61.05) vs. 71 (63.078.0) $\mathrm{mmHg}, \mathrm{P}=0.0008$, median $\mathrm{pCO}_{2} 49.6$ (47.0-51.4) vs. 43.8 (38.0-45.0) $\mathrm{mmHg}, \mathrm{P}=0.005$ at baseline and after one year, respectively] (Figure 2).

In contrast, $\mathrm{FEV}_{1}, \mathrm{VC}$ and $\mathrm{FEV}_{1} / \mathrm{VC}$ did not show a significant change $(\mathrm{P}>0.05)$ in the general population, except for the subgroup of hypercapnic patients in which there was a significant improvement in $\mathrm{FEV}_{1}$ after 1 year [70 ( -70 to 200$) \mathrm{mL}, \mathrm{P}=0.02]$. No significant variation was detected in body mass index (BMI) [31.9 (28.2-37.1) vs. 31.7 $(28.2-34.9) \mathrm{kg} / \mathrm{m}^{2}$ at baseline and after 1 year, respectively, $\mathrm{P}>0.05]$, smoking habits, or in the inhaled therapy taken, after 1 year of CPAP treatment.

Linear regression analysis revealed a significant inverse correlation between the change in $\mathrm{FEV}_{1}$ after 1 year, expressed as an absolute value, and the basal $\mathrm{FEV}_{1}$ expressed as a percentage of the predicted value (Figure 3).

A conditional inference tree-based approach identified an $\mathrm{FEV}_{1}$ baseline threshold of $79.1 \%$ of the predicted value under and above which the $\mathrm{FEV}_{1}$ change after 1 year was significantly different $\left(\mathrm{P}=1.22 \times 10^{-6}\right)$ (Figure 4). Multivariate linear regression showed that the difference observed in $\mathrm{FEV}_{1}$ variation was not influenced by potential confounders represented by age, gender and comorbidities $\left(\mathrm{P}=5.5 \times 10^{-6}\right)$.

In particular, in patients with an $\mathrm{FEV}_{1}$ at baseline below that threshold, we found a statistically significant improvement in $\mathrm{FEV}_{1}$ after 1 year of CPAP [median value 70 ( -70 to 200$) \mathrm{mL}, \mathrm{P}=0.001]$. In contrast, in patients with a baseline $\mathrm{FEV}_{1}$ above the same threshold, we observed a statistically significant decrease in $\mathrm{FEV}_{1}$ after 1 year [median value $-270(-370$ to -130$) \mathrm{mL}, \mathrm{P}=3.05 \times 10^{-5}$ ].

No difference in the median CPAP level used in patients with a basal $\mathrm{FEV}_{1}$ under or above the threshold of $79.1 \%$ was found [11 (10.0-14.0) and $11(8.5-12.5)$ respectively, $\mathrm{P}=0.489]$. Median CPAP levels according to patient's functional class are reported in more detail in Table 3.

\section{Discussion}

The main findings of this observational study of patients with OS can be summarized as follows: after 1 year of CPAP treatment, (I) ABG values significantly improved, particularly in patients with basal $\mathrm{pCO}_{2}>45 \mathrm{mmHg}$, and (II) no significant change in respiratory function emerged in the whole population. However, notably, there was (III) a significant change in $\mathrm{FEV}_{1}$ in hypercapnic patients and (IV) a significant change in $\mathrm{FEV}_{1}$ that was inversely proportional to its basal value expressed as the percentage of the predicted value $\left(\mathrm{FEV}_{1} \%\right)$ in the whole population. In particular, a lower basal $\mathrm{FEV}_{1} \%$ led to a greater increase in $\mathrm{FEV}_{1}$ after 1 year. Above a $\mathrm{FEV}_{1} \%$ of $79.1 \%$, there was an inversion of this trend.

Data on lung function changes after CPAP treatment in OS are heterogeneous in the literature. In 10 patients able to tolerate CPAP for at least 3 months, Mansfield and colleagues (15) found a significant improvement in $\mathrm{FEV}_{1}$ after a minimum of 8 months of CPAP treatment. Toraldo and colleagues (7) described an increase in $\mathrm{FEV}_{1}$ of $15 \%$ $(\mathrm{P}<0.0015)$ at 24 months compared to baseline values.

In contrast, O'Brien and Whitman (6) reported a significant decrease in $\mathrm{FEV}_{1}$ and $\mathrm{FVC}$ in overlap patients who used CPAP for more than 4 hours/day compared to non-compliant patients. Moreover, they found a strong correlation between the average daily hours of CPAP use and the percent decrease in $\mathrm{FEV}_{1}$. Similarly to O'Brien and Whitman (6), in our whole patient population, we found no differences in pulmonary function tests after 1 year of treatment. However, in contrast with previous studies, we stratified patients according to the degree of basal airflow 

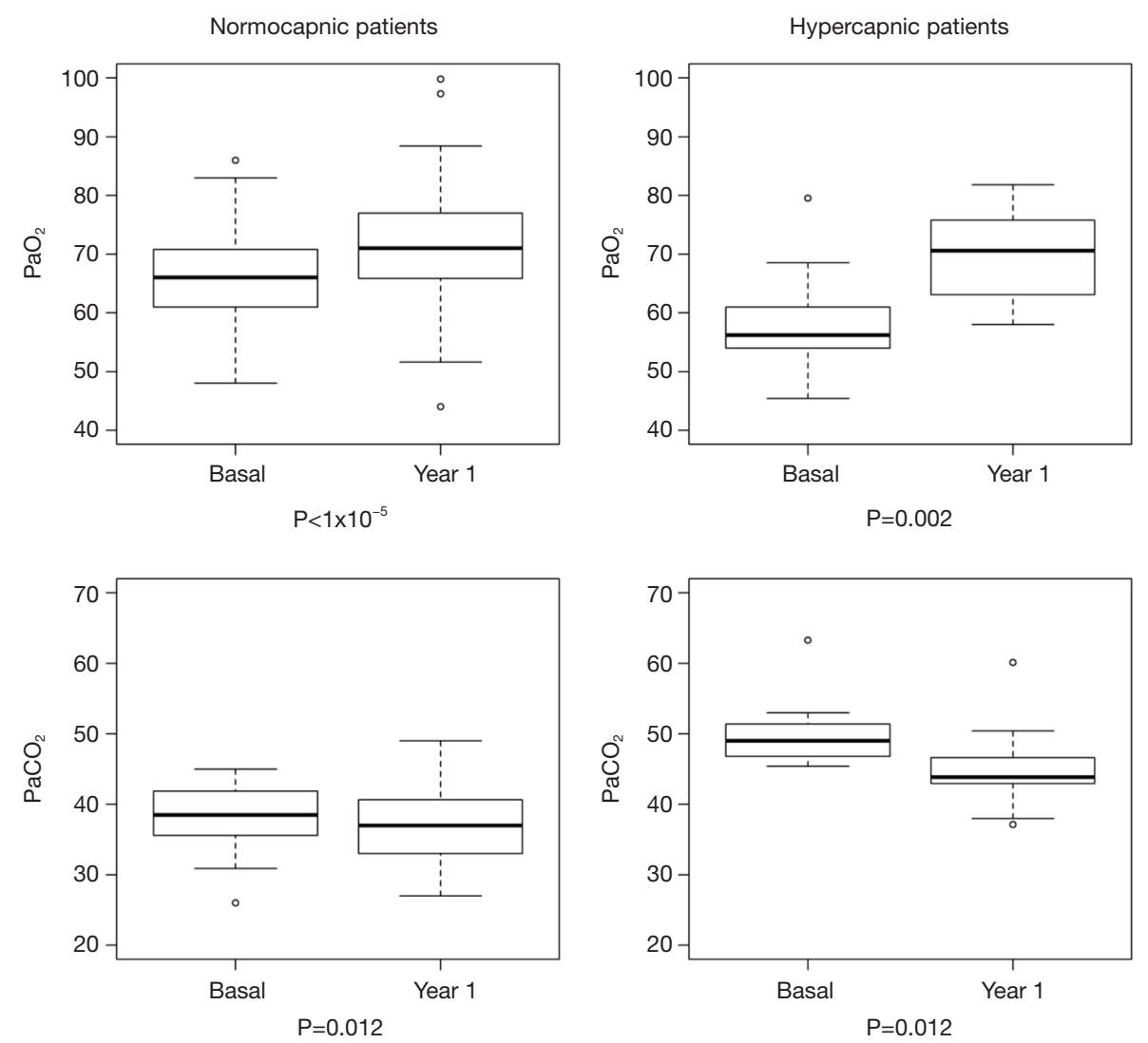

Figure $2 \mathrm{PO}_{2}$ and $\mathrm{pCO}_{2}$ changes between baseline and 1 year in normocapnic and hypercapnic patients. Both improvements appeared to be significantly greater in the subgroup of hypercapnic patients compared to the normocapnic patients [median $\mathrm{pO}_{2} 57(54.0-61.0) v s$. 71 (63.0-78.0) mmHg, $\mathrm{P}=0.0008$, median $\mathrm{pCO}_{2} 49.6$ (47.0-51.4) vs. 43.8 (38.0-45.0) mmHg, $\mathrm{P}=0.005$ at baseline and after 1 year, respectively].

obstruction. This stratification demonstrated a different effect of CPAP on respiratory function according to the basal $\mathrm{FEV}_{1} \%$ values.

Notably, the improvement and the decrease we observed under and above the $\mathrm{FEV}_{1} \%$ threshold, respectively, were independent from BMI, smoking habits and inhaled therapy variations after 1 year. They were also independent of the CPAP level used.

A possible explanation for the observed $\mathrm{FEV}_{1}$ changes could lie in the natural history of the functional decline of COPD patients which, according to recent evidence, appears to be more accelerated and more relevant in patients in the early stages than in the late stages (16). In fact, as shown by Tantucci and Modina (16), the average annual decline in $\mathrm{FEV}_{1}$ in GOLD stages II and III ranged between 47 and $79 \mathrm{~mL} /$ year, respectively, and this decline was less than $35 \mathrm{~mL} /$ year in GOLD stage IV. However, no clear data are available on the rate of decline in GOLD stage I.

Therefore, in our population, the CPAP effect may have been sufficient to overcome the natural evolution of lung function in patients with worse pulmonary function, whereas in patients in stage I with probable greater intrinsic annual functional decline, treatment may have been unable to counter the natural evolution of the disease.

A further possible explanation of our findings may lie in airway inflammation exacerbation related to OSA in COPD patients (17), which naturally affects lung function. Wang and coworkers (17) showed a significantly higher percentage of neutrophils, TNF $\alpha$ and IL-8 levels in the bronchoalveolar lavage fluid of overlap patients compared to patients with only COPD; this difference was proportional to OSA severity. The authors also found that the inflammation response significantly decreased after CPAP treatment (17). However, these data were only demonstrated in overlap patients with a moderate to severe 


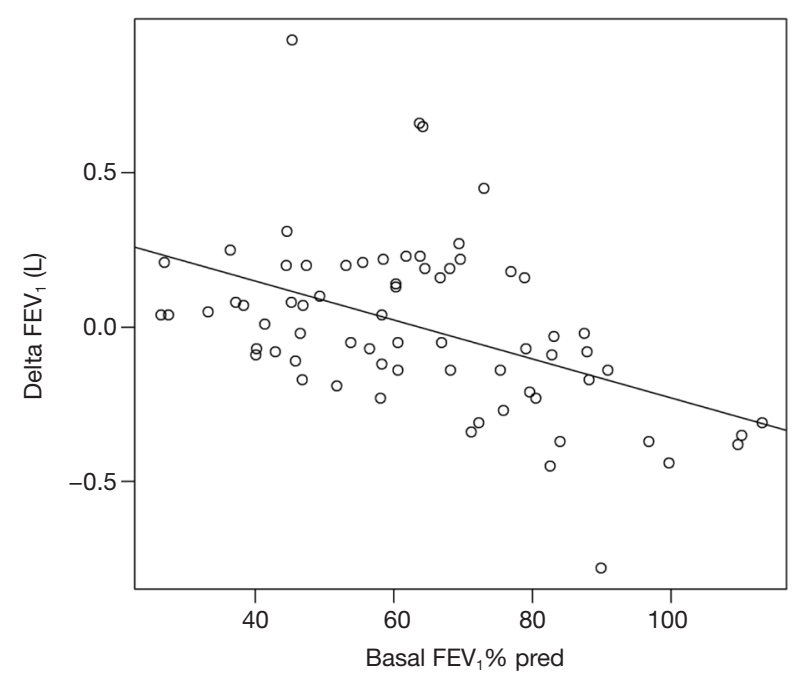

Figure 3 Correlation between change in $\mathrm{FEV}_{1}$ after 1 year and basal $\mathrm{FEV}_{1}$ expressed as the \% of the predicted value. Linear regression analysis revealed a significant inverse correlation between the change in $\mathrm{FEV}_{1}$ after one year and basal $\mathrm{FEV}_{1}$ expressed as a percentage of the predicted value emerged. $\mathrm{R}^{2}=0.79$, $\mathrm{P}<0.001 . \mathrm{FEV}_{1}$, forced expiratory volume in 1 second.

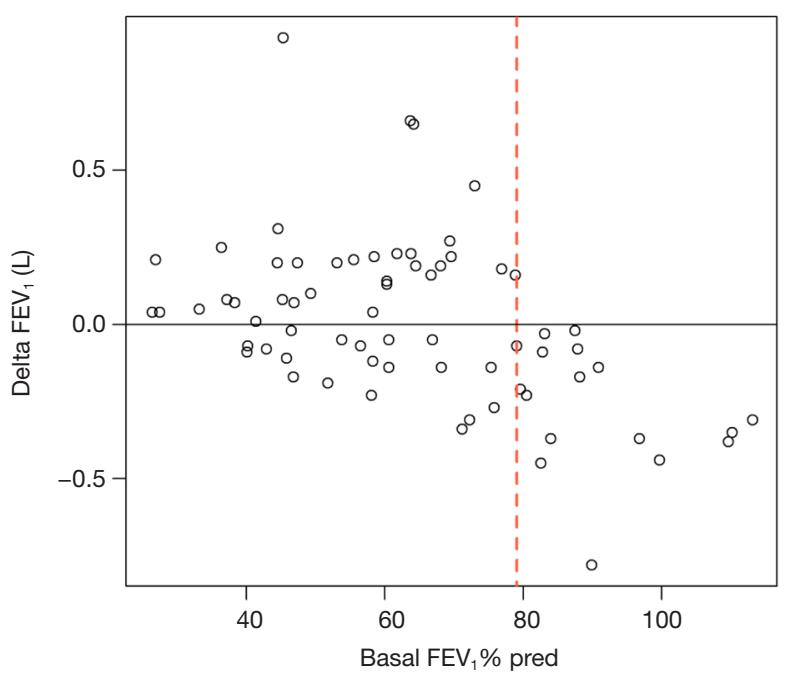

Figure 4 Threshold of $\mathrm{FEV}_{1} \%$ figure at baseline determinant of a different trend in $\mathrm{FEV}_{1}$ at 1 year. A conditional inference treebased approach identified an $\mathrm{FEV}_{1}$ baseline threshold of $79.1 \%$ of the predicted value under and above which the $\mathrm{FEV}_{1}$ change after 1 year was significantly different $\left(\mathrm{P}=1.22 \times 10^{-6}\right)$. $\mathrm{FEV}_{1}$, forced expiratory volume in 1 second.

Table 3 Median CPAP levels according to $\mathrm{FEV}_{1}$ value ( $>$ or $<$ the threshold level of $79.1 \%$ of the predicted value) and GOLD classification

\begin{tabular}{lcc}
\hline Variables & Patients $(\mathrm{n}=92), \mathrm{n}(\%)$ & CPAP level $(\mathrm{mmHg})$ \\
\hline FEV 1 (\% of predicted value) & & \\
$\leq 79.1$ & $86(93.5)$ & $11(10.0,14.0)$ \\
$>79.1$ & $6(6.5)$ & $11(8.5,12.5)$ \\
GOLD & & \\
1 & $23(25.0)$ & $11(8.2,12.7)$ \\
2 & $43(46.7)$ & $12(10.0,14.0)$ \\
3 & $20(21.7)$ & $11(10.0,13.2)$ \\
4 & $6(6.5)$ & $12(12.0,13.0)$ \\
\hline
\end{tabular}

CPAP, continuous positive airway pressure; $\mathrm{FEV}_{1}$, forced expiratory volume in 1 second; GOLD, Global Initiative for Chronic Obstructive Lung Disease.

functional defect. No data on the level of inflammation induced by OSA in patients with mild COPD are available. Therefore, we may hypothesize that a lower level of airway inflammation in patients with mild COPD and OSA could explain the absence of a positive effect of CPAP on respiratory function.

In line with previous studies, we found an improvement in ABGs after 1 year of CPAP treatment. In the study by de Miguel et al. (8), statistically significant increases in $\mathrm{PaO}_{2}$ and decreases in $\mathrm{PaCO}_{2}$ were observed 6 months after starting CPAP therapy, and the improvement in ABGs was greater in the group of patients with hypercapnia at baseline. Mansfield et al. (15) also found a significant improvement in ABGs in patients who were compliant with nasal CPAP.

In our study, hypercapnic patients appeared to benefit 
more from the ventilator treatment than the normocapnic patients, both in terms of gas exchanges and respiratory function.

This result underlines an important issue in the clinical practice: hypercapnic respiratory failure in COPD patients must be investigated for the presence of OSA, and a first attempt at using CPAP therapy should be made.

The improvements in function and $\mathrm{ABG}$ may be related to some CPAP effects. In fact, CPAP can reduce inspiratory effort during sleep in patients with COPD, as documented by significant reductions in the tidal excursions of esophageal pressure (18). Furthermore, CPAP may lead to an improvement in the ventilation/perfusion ratio. In fact, by correcting obstructive events and limiting the consequent increase in the work of breathing, CPAP acts on alveolar hypoventilation and corrects nocturnal hypoxemia and hypercapnia. The reversal of nocturnal blood gases and the resetting of chemoreceptor sensitivity are then responsible for the improvements in diurnal ABGs (19). Finally, the normalization of arterial $\mathrm{pCO}_{2}$ in patients who were hypercapnic at baseline may reduce intramural bronchial edema, which may be determined by hypercapnia-induced renal vasoconstriction (20). This may explain the significant improvement of $\mathrm{FEV}_{1}$ in the subgroup of hypercapnic overlap patients.

Our study has some limitations. Firstly, this is a retrospective study, and data on body plethysmography at 1 year are thus not available for all patients.

In addition, data concerning acute exacerbations of COPD are lacking, and therefore the effect of CPAP treatment on their incidence in the different functional stages is also unknown. As we surmised that the improvement in pulmonary function may be related to a decrease in the number of exacerbations, a prospective study exploring this issue could be interesting.

Regarding the limited sample size potentially affecting this study, post hoc calculations were performed to estimate the statistical power reached for the variations observed in terms of $\mathrm{pO}_{2}$ and $\mathrm{pCO}_{2}$ from baseline to year 1 measurements. Calculations were also performed to assess the differences in terms of FEV1 variations between subjects with baseline $\mathrm{FEV}_{1} \leq 79.1 \%$ and the rest of the cohort. The findings provide reassurance of the robustness of these results, showing sufficient statistical power $(\geq 0.96)$ for the three tests.

Finally, the follow-up of our patients lasted only 1 year, as data on the subsequent years are lacking. It would have been interesting to continue to monitor both patients, with functional improvements and those with functional losses, to gain insights into their functional evolution.

\section{Conclusions}

In conclusion, our retrospective study of patients affected with OSA and COPD found that despite a significant improvement in gas exchanges in the whole population, after 1 year of CPAP treatment the pattern of respiratory function was different according to the basal degree of airway obstruction. In patients with an $\mathrm{FEV}_{1}$ lower than $79.1 \%$, there was a significant improvement in $\mathrm{FEV}_{1}$ that was inversely proportional to the basal value, whereas patients with an $\mathrm{FEV}_{1}$ higher than $79.1 \%$ experienced a significant worsening of their respiratory function. Further observational studies are needed to confirm these findings.

\section{Acknowledgements}

None.

\section{Footnote}

Conflicts of Interest: The authors have no conflicts of interest to declare.

Ethical Statement: The study was approved by the Ethics Committee of the Istituti Clinici Scientifici Maugeri (Number 844 CEC) and written informed consent was obtained from all patients.

\section{References}

1. Lee R, McNicholas WT. Obstructive sleep apnea in chronic obstructive pulmonary disease patients. Curr Opin Pulm Med 2011;17:79-83.

2. Flenley DC. Sleep in chronic obstructive lung disease. Clin Chest Med 1985;6:651-61.

3. Ioachimescu OC, Teodorescu M. Integrating the overlap of obstructive lung disease and obstructive sleep apnoea: OLDOSA syndrome. Respirology 2013;18:421-31.

4. Owens RL, Malhotra A. Sleep-Disordered Breathing and COPD: The Overlap Syndrome. Respir Care 2010;55:1333-44; discussion 1344-6.

5. Marin JM, Soriano JB, Carrizo SJ, et al. Outcomes in patients with chronic obstructive pulmonary disease and obstructive sleep apnea: the overlap syndrome. Am J Respir Crit Care Med 2010;182:325-31. 
6. O'Brien A, Whitman K. Lack of Benefit of Continuous Positive Airway Pressure on Lung Function in Patients with Overlap Syndrome. Lung 2005;183:389-404.

7. Toraldo DM, De Nuccio F, Nicolardi G. Fixed-pressure nCPAP in patients with obstructive sleep apnea (OSA) syndrome and chronic obstructive pulmonary disease (COPD): a 24-month follow-up study. Sleep Breath 2010;14:115-23.

8. de Miguel J, Cabello J, Sánchez-Alarcos JM, et al. LongTerm Effects of Treatment with Nasal Continuous Positive Airway Pressure on Lung Function in Patients with Overlap Syndrome. Sleep Breath 2002;6:3-10.

9. Wanger J, Clausen JL, Coates A, et al. Standardisation of the measurement of lung volumes. Eur Respir J 2005;26:511-22.

10. Miller MR, Hankinson J, Brusasco V, et al. Standardisation of spirometry. Eur Respir J 2005;26:319-38.

11. Vogelmeier CF, Criner GJ, Martinez FJ, et al. Global Initiative for Chronic Obstructive Lung Disease Global strategy for the diagnosis, management, and prevention of chronic obstructive pulmonary disease 2017 Report: GOLD executive summary. Am J Respir Crit Care Med 2017;195:557-82.

12. American Academy of Sleep Medicine. International Classification of Sleep Disorders 2nd edition: Diagnostic and coding manual. American Academy of Sleep Medicine, 2005. American Academy of Sleep Medicine International Classification of Sleep Disorders 3rd edition. Darien, IL: American Academy of Sleep Medicine, 2014.

13. Berry RB, Brooks R, Gamaldo CE, et al. for the American Academy of Sleep Medicine. The AASM Manual for

Cite this article as: Schreiber A, Surbone S, Malovini A, Mancini M, Cemmi F, Piaggi G, Ceriana P, Carlucci A. The effect of continuous positive airway pressure on pulmonary function may depend on the basal level of forced expiratory volume in 1 second. J Thorac Dis 2018;10(12):6819-6827. doi: 10.21037/jtd.2018.10.103 the Scoring of Sleep and Associated Events: Rules, Terminology and Technical Specifications. Version 2.4. Darien, IL: American Academy of Sleep Medicine, 2017.

14. Kushida CA, Chediak A, Berry RB, et al. Positive Airway Pressure Titrsation Task Force; American Academy of Sleep Medicine. Clinical Guidelines for the manual titration of positive airway pressure in patients with obstructive sleep apnea. J Clin Sleep Med 2008;4:157-71.

15. Mansfield D, Naughton MT. Effects of continuous positive airway pressure on lung function in patients with chronic obstructive pulmonary disease and sleep disordered breathing. Respirology 1999;4:365-70.

16. Tantucci C, Modina D. Lung function decline in COPD. Int J Chron Obstruct Pulmon Dis 2012;7:95-9.

17. Wang Y, Hu K, Liu K, et al. Obstructive sleep apnea exacerbates airway inflammation in patients with chronic obstructive pulmonary disease. Sleep Med 2015;16:1123-30.

18. Petrof BJ, Kimoff RJ, Levy RD, et al. Nasal continuous positive airway pressure facilitates respiratory muscle function during sleep in severe chronic obstructive pulmonary disease. Am Rev Respir Dis 1991;143:928-35.

19. Rossi A, Santos C, Roca J, et al. Effects of PEEP on VA/Q mismatching in ventilated patients with chronic airflow obstruction. Am J Respir Crit Care Med 1994;149:1077-84.

20. Hemlin M, Ljungman S, Carlson J, et al. The effects of hypoxia and hypercapnia on renal and heart function, haemodynamics and plasma hormone levels in stable COPD patients. Clin Respir J 2007;1:80-90. 